Voix et Images

\title{
Les Manuscrits de Pauline Archange de M.-C. Blais : Éros et Thanatos
}

\section{Alain-Bernard Marchand}

Volume 7, numéro 2, hiver 1982

Michel Tremblay

URI : https://id.erudit.org/iderudit/200324ar

DOI : https://doi.org/10.7202/200324ar

Aller au sommaire du numéro

Éditeur(s)

Les Presses de l'Université du Québec

\section{ISSN}

0318-9201 (imprimé)

1705-933X (numérique)

Découvrir la revue

\section{Citer cet article}

Marchand, A.-B. (1982). Les Manuscrits de Pauline Archange de M.-C. Blais : Éros et Thanatos. Voix et Images, 7(2), 343-349. https://doi.org/10.7202/200324ar d'utilisation que vous pouvez consulter en ligne.

https://apropos.erudit.org/fr/usagers/politique-dutilisation/ 


\title{
Les Manuscrits de Pauline Archange de M.-C. Blais : Éros et Thanatos
}

\author{
par Alain-Bernard Marchand
}

"Mais qu'est-ce que l'enfer? Est-ce ce bourdonnement fou de la peur à mon oreille quand je crois entendre gémir les damnes, dans le silence du dortoir, ou la crainte d'un châtiment plus obscur sur la terre?" (Manuscrits, p. 59).

Éros et Thanatos, forces perpétuellement insatisfaites et inquiètes, s'inscrivent dans Les Manuscrits de Pauline Archange comme deux pôles d'attraction entre lesquels s'insère la mise en forme d'une expérience vouée à l'enfer de la souffrance absurde. A la fois puissance générale d'unification et figure destructive de l'existence, l'écriture blaisienne est, à tous les niveaux, le lieu de rencontre ad partes où coexistent la vie et la mort, c'est-à-dire une tension entre des forces contraires dont les éléments sont en constante fluctuation. En tant que symbole, Thanatos nous introduit d'emblée dans le monde inconnu des enfers, un "monde étant toujours à feu et à sang" ', avant de nous ouvrir l'accès à une vie nouvelle où se délivrent les forces négatives et régressives par le seul renouvellement de la forme qui est elle-même recréée par la déflagration du langage. A l'instar du jeune héros au front couronné de poux d'une Saison, pendant masculin de l'héroïne des Manuscrits, l'Archange Pauline incarne une pulsion créatrice qui se déploie au contact d'une réalité sordide et reconstitue un univers à la fois décomposé et recomposé où règnent seules les contradictions humaines : “le noir et le tendre "2, Thanatos, pulsion de mort, et Éros, pulsion de vie, tout ensemble destruction et création suprêmes.

Tout comme son prédécesseur Jean-le-Maigre dont elle a hérité la supra-lucidité, Pauline Archange se découvre en bas âge au sein même d'une réalité ambiante teintée de macabre où baignent des personnages insolites condamnés à la décrépitude la plus opiniâtre. Seule devant l'atrocité bestiale et le silence déraisonné du monde, Pauline Archange est à la fois le "gladiateur" ${ }^{3}$ et le "lion" 4 ; celle qui apprivoise durement ses semblables, “tortur(ant) les autres afin d'éviter les mêmes tortures pour soi " 5 , celle qui refuse de se vautrer dans l'ignominie d'une pareille existence à grand renfort de révolte. À peine commence-t-elle à explorer le vaste 
monde que cette super-fillette est aux prises avec l'obscurité pénétrante de l'extérieur qui semble porter la couleur fatale. Songeons particulièrement à "cet endroit sans lumière " 6 où se rassemble la Compagnie des Mireillettes dont Pauline fait partie ainsi qu'aux "salles obscures des classes faiblement éclairées par les ampoules électriques " 7 . Bien plus qu'une atmosphère, le noir s'impose avec force fougue et se métamorphose en une puissance mystérieuse, formant ainsi un microcosme thématique à l'intérieur de ce macrocosme miséreux où «la vie n'est qu'un souffle qui peut vous quitter à tout instant " 8 . Se mouvant comme "un fantôme parmi d'autres fantômes ${ }^{9}$ sous les épaisseurs opprimantes de cet enfer dantesque, Pauline Archange, au-delà de la révolte, cherche à réinventer un univers à partir de cette tabula rasa afin de reconquérir son identité. "Dans une indépendance farouche, écrit-elle, nous écartions les liens du sang pour renaître à notre façon intime, d'une naissance spiritualisée où les parents cette fois ne joueraient plus aucun rôle, laissant à nos nombreux désirs une existence à remplir, un paysage désert à habiter " ${ }^{10}$.

En effet, même la cellule familiale, "ce tribunal fermé»"11, cette "table de calomnie" 12 , impose à l'enfance une cruelle et monstrueuse autorité contre laquelle Pauline Archange s'insurge héroïquement. Cet univers peuplé d'enfants permet à Marie-Claire Blais de transgresser les règles sociales afin de percer le voile mensonger des apparences, séparant ainsi la réalité brute de la réalité préfabriquée. Entre une mère «perdue si loin dans le brouillard austère de sa vie" ${ }^{13}$, un père «las et affamé après ses journées à l'usine »14, les religieuses, "chceur de fauves amers» ${ }^{15}$, Pauline Archange, quoiqu'un "peu moins médiocre que les autres" ${ }^{16}$ grâce à sa précocité d'esprit, se sent «lasse comme mille hommes et brisée comme une armée " 17. Comme pour les Enfants terribles de Cocteau, un système de valeurs décadent hante le cœur et l'esprit de Pauline Archange et lui inspire une indignation effrénée qui se manifeste même dans ses rêves, "visions sanglantes " ${ }^{18}$, qui ont naturellement tendance à dégénérer en cauchemars. II s'agit donc ici, comme le remarque Paule Leduc, d'une enfance qui n'en est pas une, une enfance où les lois, celles de la violence de l'être, de la violence de la liberté, reconduisent l'être au monde de la finitude, "au monde de l'adulte " ${ }^{19}$. C'est une spirale sans fin, qui entraîne dans son tourbillon les illusions et "l'indépendance» ${ }^{20}$ et grâce à laquelle Marie-Claire Blais, par le biais de la création, Éros, dénonce sans vergogne la prépondérance de Thanatos au cœur d'une société mi-cauchemardesque, mi-infernale.

Cette dénonciation s'effectue sciemment par le biais d'une narratrice qui se dédouble en une Pauline Archange qui vit et une Pauline Archange qui écrit, Thanatos et Éros, instinct de destruction et instinct de création. On peut ainsi remarquer dans ce roman un phénomène de schizophrénie littéraire; c'est-à-dire que l'héroïne, à la fois affrontée et absorbée par l'absurdité de son existence, recherche par le truchement de l'écriture le secret de sa propre image. L'écriture, insérée entre ces deux niveaux de conscience artistement mis en rapport par la romancière, devient à la 
limite la véritable voie vers la découverte de l'identité. Ce sont les mots euxmêmes qui forment l'écran derrière lequel se dérobe le visage que l'on croit apercevoir dans le silence d'une nuit omnipresente. La seule loi de Pauline Archange est celle de la «Découverte», de l'autodécouverte de soi et de l'autre par l'incessante confrontation avec la violence de la nature et des éléments, par l'insatiable besoin de dépasser le gouffre de médiocrité bestiale la séparant du monde des adultes. Pauline Archange exprime clairement son désarroi et sa révolte : «(..) la nature, perfide, l'absurdité de la vie ouvrant sous nos pieds des traces noires et vides dans lesquelles tournoyaient les grandes personnes, comme des insectes avides à la faible lumière de leur dégout, buvant, mangeant, prononçant des paroles suspectes et se reproduisant sans fin pour leur propre malheur" 21. Ainsi, ce monde adulte, inquisiteur, qu'il faut oublier pour le mieux détruire, est à la fois la cible et le but de l'enfance ${ }^{22}$.

Cet inquiétant univers extérieur dans lequel la romancière fait merveilleusement mariner toute une galerie onirique de personnages à la fois lugubres et attachants n'est pas sans rappeler le délire grandiose de Fellini. Par le seul mouvement de sa plume, Marie-Claire Blais fait déferler sous nos yeux ébahis tout un cortège de fauves rebelles et de monstres féroces; dans ce musée imaginaire s'anime une fresque sur laquelle apparaissent à tour de rôle Séraphine, enfant nerveusement soumise, "plus victime que nos petites saintes sur les images "23, finalement "broyée sous les roues de l'un de ces autobus aveugles" 24 ; la mère d'Huguette Poire «qui port(e) le costume de ses songes du matin au soir» ${ }^{25}$, son mari "s'adonn(ant) à tout un concert surpris qui montait de son estomac à sa gorge » 26 et, enfin, tout un serpentin d'enfants pâles «encerclés dans leur maladie comme d'une condition de vivre née avec eux " 27 ; I'oncle Sébastien dont on sent le "sourire souffrant» 28 et le «délire courroucé de son regard " 29 ; au bout d'une «petite route où s'achevait le monde» ${ }^{30}$, SaintOnge-du-Délire, là où d' "âpres figures" 31 "mange(nt) le pain noir, les raisins noirs " ${ }^{32}$; le frêle Jacob, faisant partie de "la caste avilie des infirmes " 33 , dont les rêves sont hantés par «des géants avec des dents noires et des yeux bleus pleins de crachats" 34 ; des couvents aux sombres corridors où déambulent “ des araignées noires avec des p'tites pattes jaunes " ${ }^{35}$, dissimulant derrière leurs longues robes une sensualité réprimée, et où il faut éviter de se toucher des coudes car "c'est péché "36; Louisette Denis qui sait "hurler comme un diable qu'on brâle par la queue" 37 si l'on ose attaquer sa liberté; d'étranges «héros qui n'ont pas peur d'éteindre la flamme partout où elle brûle et consume, où elle ravage et tue "38 et qui affirment que «l'enfer est ici même" ${ }^{39}$. Bref, tout un amas "d'agonisants qu'il eût été préférable d'enterrer tout de suite ${ }^{40}$ et sur lesquels surplombe "l'œil de Dieu brillant de malice et de dureté " ${ }^{41}$.

Mais à l'intérieur de cet attirail concerté de monstruosités fantasques, Éros, pulsion de création, semble la seule forme de récupération possible du mal, Thanatos. L'inquiétude et le vertige qu'inspire la lecture de ce 
roman-fresque naissent du double mouvement contradictoire : attrait, répulsion. Par le biais du processus créatif, la romancière évoque paradoxalement une sorte d'antichambre de l'Apocalypse où la misère immonde s'exalte en maîtresse dictatoriale, affectant à la fois la chair et l'esprit. Comme dans une Saison, la misère physique: faim, froid et mauvais traitements règnent avec force. Cette misère physique rattache les personnages à un monde animal, au niveau de la brute dépourvue d'âme. Harcelée par la rigueur du vent et de la neige "qui (lui) frappent brusquement les tempes 42 lors de ses promenades, Pauline Archange s'obstine aussi à ne pas vouloir manger et s'évanouit fréquemment pendant les classes. Elle subit également la férocité outrée du père de Jacob par un des "soirs de grands fouets " ${ }^{43}$. La misère physique entraîne inévitablement la douleur morale fanatiquement convoitée par les faux préjugés de l'Église supposée justicière qui, en revanche, engendre un climat d'obscurantisme propice à sa souveraineté. Tout comme elle l'a fait pour la famille, Marie-Claire Blais démystifie, à force d'ironie et de parodie, les portraits traditionnels du prêtre et de la religieuse ici représentés de façon caricaturale. On songe tout de suite à l'aumônier ennuyé par "toutes ces femmes qui avaient tourné autour de lui comme des mouches " 44 et à “l'extase pétrifiée " 45 des religieuses dont "(les) petits yeux cour(ent) comme des billes dans la lune contemplative de leur visage ${ }^{46}$. Par le soudain écart par rapport à la réalité brute, Thanatos, noirceur forcenee, prend quelques-unes des couleurs d'Éros, - et cela, grâce au seul processus de la création qui transforme un univers hideux en une œuvre d'art où forme et fond se conjuguent pour conduire à l'émerveillement.

L'univers magique des Manuscrits, "ce jardin étrange où pouss(ent), là comme ailleurs, entremêlent leurs tiges, les plantes gracieuses du Vice et de la Vertu » ${ }^{47}$, n'est pas pour autant sauvegardé d'un certain pessimisme qui afflue ad libitum sous la poussée créatrice d'Éros. L'éclosion d'une telle géhenne où "chacun (...) (est) seul, capable à chaque instant d'un monstrueux oubli à l'égard de son frère, ivre de soi jusqu'au crime " 48 , incite Pauline Archange à ne voir que le mal tellement il est omniprésent; tout semble inapte dans le domaine de la tendresse sinon les quelques amitiés éphémères qui dégénèrent toujours en un "combat " 49 , celui de "deux solitudes qui ne se rencontr(ent) plus "50. Chacun est ainsi renvoyé à soi, dans sa nuit essentielle, enchaîné à ces lieux macabres où il faut «toujours vivre en soi-même comme dans une prison" ${ }^{51}$, là où les êtres se "parl(ent) sans (se) voir, chacun (d'eux) préservant son secret en tournant le dos à l'autre "52. À cette incommunicabilité se rattache l'avidité humaine qui pénètre partout, "et à son secours ne (vient) aucune compassion égale à ses besoins" ${ }^{3}$. La terre, selon sa représentation ab absurbo, devient "le royaume de l'oubli»54, "un accablant lieu de délices d'où la charité serait exclue»55, où l'amour semble absurde "quand tant d'hommes (meurent) à chaque instant " 56 et la mort "une vengeance éternelle " 57 . II va donc sans dire que les esprits nus, "cette cire humaine (...) sans enveloppe protectrice contre le monde " ${ }^{58}$, sont d'ores et déjà voués à une dissolution indéniable 
face à cette réalité où la frontière commune entre l'homme et l'extérieur ambiant porte le sceau fatal de Thanatos. À la fois séparé des autres et fébrilement revêtu d'un "manteau de vertu que l'on (jette) sur sa vraie nature " ${ }^{59}$, on s'éloigne aussi de soi, "(pris) dans (sa) dignité comme dans un corset de fer» ${ }^{60}$. On se recherche dans "ces miroirs estompés qui (nous) reflètent avec mensonge ${ }^{61}$ et l'on finit par devenir étranger "à sa propre image»62, subissant avec horreur «la persécution d'être (vu)»63. Cette résignation stoïque devant une telle condition absurde, "ce long silence "64, «deviendrait-il un jour la rédemption des hommes?»65 se demande Pauline Archange qui doit tirer de "ces vivants et ces morts dégénérés (...), plus que la naissance, plus que la vie, (sa) résurrection ${ }^{66}$.

Mais cet épanchement quasi obsessionnel dans les misères immondes de la chair et de l'esprit se trouve en retour atténué par le décalage maintenu entre la réalité brute, Thanatos, et la réalité romanesque, Éros, s'exprimant dans le récit par l'inaltérable “on" anonyme, à la fois pronom personnel et indéfini, dont la portée illimitée insuffle à l'œuvre une touche certaine d'universalité. Le 'on' marque aussi la charnière entre les deux niveaux de conscience perçus dans le roman : Pauline Archange qui vit et Pauline Archange qui écrit. A ces deux niveaux de conscience s'associent deux espaces temporels à l'intérieur des Manuscrits : le temps chronologique qui couvre une période approximative de quatre ans et le temps de l'écriture quii semble se développer au rythme sinueux de la mémoire narratrice. Cette dualité sur le plan de l'écriture nous rappelle que le monde est constitué de fragments, et que toute vision de ce monde est subjective. "Créer, c'est vivre deux fois " 67 disait Camus. Dans un tel univers où foisonnent des myriades d'impressions, les êtres, l'écriture et le temps sont discontinus par nature, et le roman doit surtout mettre en lumière cette discontinuité imprévisible. Les Manuscrits de Pauline Archange exprime assez bien ce va-et-vient constant entre le dehors et le dedans, cette alternance de perception et de réflexivité qui font que Thanatos se retrouve étonnamment transformé en Éros, lente métamorphose qui mène de la destruction à la création.

Par le biais de la narration, cette super-fillette « née dans le récit même qu'(elle) voul(ait) écrire "68 provoque une espèce d'exorcisme de la pensée : l'anarchie des formes romanesques qu'elle utilise avec force brio lui permet d'ériger un univers où il fallait "seulement des dents pour mordre " ${ }^{69}$ en une vision où la fermentation de l'esprit s'unit à celle de la forme littéraire. En effet, dans les Manuscrits, l'agir ne semble pas précéder l'écriture, mais l'accomplit, tellement les deux sont bien amalgamés à l'intérieur de ce macrocosme romanesque; formant un étroit plexus indissociable, l'agir et l'écriture libèrent les forces destructives et créatrices, Thanatos et Éros, et se cristallisent au sein même de l'œuvre sans laquelle Pauline Archange “risqu(ait) de n'avoir existé pour personne» 70 . Créer, comme le dit Camus, c'est ainsi donner une forme à son destin ${ }^{71}$. Au tréfonds de cet abîme infernal où "l'innocence (est) la mort" ${ }^{72}$ et le mal la 
vie, "l'imagination (écrit) de fougueux récits pendant que le corps (feint) de dormir" 73 , Eros à l'assaut de Thanatos, tout ensemble destruction et création suprêmes, seul espoir d'échapper à l'enfer de la souffrance absurde.

Ainsi, sous l'outrecuidante morsure de la prose poétisée de MarieClaire Blais, catharsis allègrement opiacée d'aigre-douceur, se lovent les deux forces extrêmes, Éros et Thanatos, incantations symboliques s'inventant au fil de la trame romanesque, sublime expurgation de la chair et de l'esprit enfin réunis au sein de cette cosmographie qui, par-delà sa propre existence, fusionne à cor et à cri les contradictions absurdes de l'expérience créatrice. Destruction et création, instinct de mort et instinct de vie, Thanatos et Éros, faisant litière de toute aversion, ne se recherchent plus dans les Manuscrits de Pauline Archange comme des contraires, mais comme deux entités omniprésentes et inséparables.

1. Marie-Claire Blais, Manuscrits de Pauline Archange, Paris, Les Editions Grasset, 1968, p. 53.

2. Vincent Nadeau, Marie-Claire Blais : le noir et le tendre, Montréal, Les Presses de l'Université de Montréal, 1974, Titre de la thèse.

3. Blais, op. cit., p. 124.

4. Ibid.

5. Ibid., p. 187.

6. Ibid., p. 57.

7. Ibid., p. 133.

8. Ibid., p. 166.

9. Ibid., p. 114.

10. Ibid., p. 23.

11. Ibid., p. 24.

12. Ibid.

13. Ibid., p. 34.

14. Ibid., p. 112.

15. Ibid., p. 66 .

16. Ibid., p. 185.

17. Ibid., p. 90.

18. Ibid., p. 53.

19. Paule Leduc, "Le roman (1968) », Études Littéraires, vol. 2, no 2, aoât 1969, p. 208.

20. Ibid.

21. Blais, p. 21.

22. Op. cit., p. 207.

23. Blais, p. 15.

24. Ibid., p. 61.

25. Ibid., p. 27.

26. Ibid., p. 32.

27. Ibid., p. 38.

28. Ibid., p. 52.

29. Ibid.

30. Ibid., p. 73.

31. Ibid., p. 74.

32. Ibid., p. 72.

33. Ibid., p. 75 . 
34. Ibid., p. 77.

35. Ibid., p. 98.

36. Ibid., p. 160.

37. Ibid., p. 92.

38. Ibid., p. 203.

39. Ibid

40. Ibid., p. 36.

41. Ibid., p. 18.

42. Ibid., p. 11.

43. Ibid., p. 83.

44. Ibid., p. 102.

45. Ibid., p. 12.

46. Ibid., p. 9.

47. Marie-Claire Blais, Une saison dans la vie d'Emmanuel, Montréal, Les Éditions du Jour, 1970, p. 49.

48. Blais, Manuscrits, p. 64.

49. Ibid.

50. Ibid.

51. Ibid., p. 186.

52. Ibid., p. 42.

53. Ibid., p. 65.

54. Ibid.

55. Ibid.

56. Ibid., p. 53.

57. Ibid., p. 63.

58. Ibid., p. 115.

59. Ibid., p. 141.

60. Ibid., pp. 122-3.

61. Ibid., p. 67.

62. Ibid.

63. Ibid., p. 89.

64. Ibid., p. 68.

65. Ibid.

66. Ibid., p. 182.

67. Albert Camus, Le mythe de sisyphe, Saint-Amand, Gallimard, 1973, p. 128.

68. Blais, Manuscrits, p. 206.

69. Ibid., p. 83.

70. Ibid., p. 206.

71. Camus, op. cit., p. 156.

72. Ibid., p. 179.

73. Ibid., p. 152. 